\title{
Analysis of Low-Speed Stall Aerodynamics of a Swept Wing with Seamless Flaps
}

\author{
Trong T. Bui ${ }^{1}$ \\ NASA Armstrong Flight Research Center, Edwards, California 93523
}

\begin{abstract}
Computational fluid dynamics (CFD) analysis was conducted to study the low-speed stall aerodynamics of a Gulfstream G-III airplane (Gulfstream Aerospace Corporation, Savannah, Georgia) swept wing modified with an experimental seamless, compliant flap called the Adaptive Compliant Trailing Edge (ACTE) flap. The stall characteristics of the modified ACTE wing were analyzed and compared with the unmodified, clean wing at the flight speed of $120 \mathrm{kn}$ and altitude of $2300 \mathrm{ft}$ above mean sea level, in free air as well as in ground effect. A polyhedral finite-volume unstructured full Navier-Stokes CFD code, STAR-CCM+ ${ }^{\circledR}$ (CD-adapco [Computational Dynamics Limited, United Kingdom, and Analysis \& Design Application Co., United States]), was used. Steady Reynolds-averaged Navier-Stokes CFD simulations were conducted for a clean wing and the ACTE wings at various ACTE deflection angles in free air (-2 deg, $15 \mathrm{deg}$, and $30 \mathrm{deg}$ ) as well as in ground effect (15 deg and $30 \mathrm{deg})$. Solution sensitivities to grid densities were examined. In free air, the ACTE wings are predicted to stall at lower angles of attack than the clean wing. In ground effect, all wings are predicted to stall at lower angles of attack than the corresponding wings in free air. Even though the lift curves are higher in ground effect than in free air, the maximum lift coefficients for all wings are lower in ground effect. Finally, the lift increase due to ground effect for the ACTE wing is predicted to be less than the clean wing.
\end{abstract}

\section{Nomenclature}

ACTE $=$ Adaptive Compliant Trailing Edge

AFRC $=$ Armstrong Flight Research Center

AIAA $=$ American Institute of Aeronautics and Astronautics

AoA $=$ angle of attack

ASL $\quad=$ above sea level

$\mathrm{C}_{\mathrm{L}} \quad=$ coefficient of lift

$\mathrm{CFD}=$ computational fluid dynamics

NASA $=$ National Aeronautics and Space Administration

SCRAT $=$ SubsoniC Research AircrafT

\section{Introduction}

$\mathrm{T}$ HE National Aeronautics and Space Administration (NASA) Armstrong Flight Research Center (Edwards, California) (AFRC) SubsoniC Research AircrafT (SCRAT) tail number $804,{ }^{1}$ is a modified Gulfstream III (G-III) (Gulfstream Aerospace Corporation, Savannah, Georgia), that is used as the flight demonstrator for the Adaptive Compliant Trailing Edge (ACTE) Project. As a joint effort between NASA, the U.S. Air Force Research Laboratory, and FlexSys Inc. (Ann Arbor, Michigan), the ACTE flight project demonstrates an experimental adaptive compliant flap in flight. ${ }^{2}$ The ACTE flap is a variable-geometry trailing edge device that is seamless to the surrounding area of the wing of the airplane. The ACTE flap technology has been tested in small-scale flight tests. ${ }^{3}$ The NASA AFRC

\footnotetext{
${ }^{1}$ Aerospace Engineer, Aerodynamics and Propulsion Branch, P.O. Box 273, M.S. 2228, Senior Member AIAA.
} 
SCRAT expands the flight research of the ACTE flap technology to the higher flight loads, Reynolds numbers, and Mach numbers that are representative of typical full-scale jetliners.

Hereafter with ACTE flaps installed, the NASA G-III SCRAT aircraft is called the ACTE aircraft, and the G-III SCRAT wing is called ACTE wing. The ACTE aircraft with the ACTE flap at a deflection of 30 deg, for example, is called the 30-deg ACTE aircraft. Figure 1 shows a solid computer-aided design (CAD) model of the 30-deg ACTE aircraft. To distinguish various parts, different parts of the aircraft have different colors. For example, the ACTE main flap section on the left wing is light tan. The inboard transition section is orange, and the outboard transition section is light blue. As shown in this figure, the ACTE flaps replace the conventional aircraft flaps on each wing of the ACTE aircraft. The ACTE flap spans approximately 50 percent of the wing half-span and approximately 20 percent of the wing chord, fully occupying the entire cove of the original flap. The inboard and outboard transition sections provide seamless connections to the surrounding wing surfaces. In addition to replacing the original flaps, the ACTE flap installation required the removal of the G-III aircraft's ground and flight spoilers from each wings. For this project, the ACTE flap does not move in-flight. Rather, the deflection of the flap is fixed at a predetermined angle before each flight and remains fixed in that position from take-off to landing.

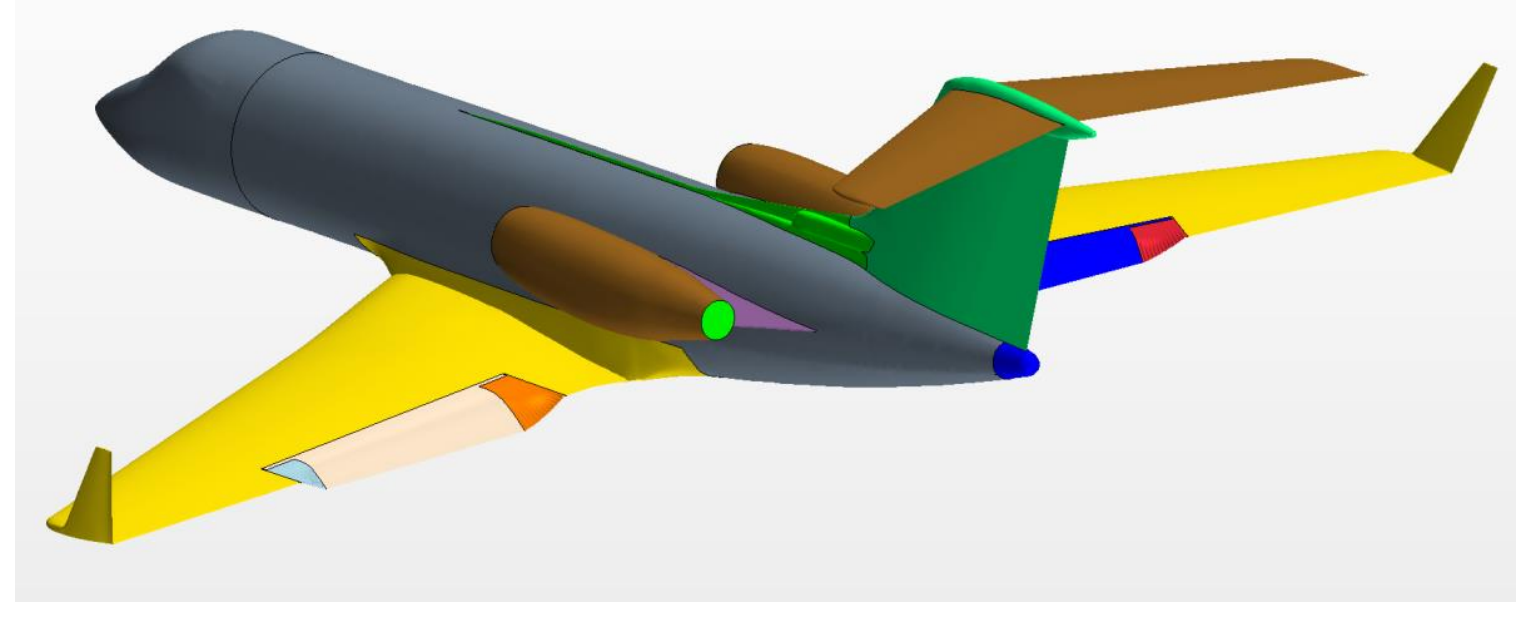

Figure 1. Solid computer-aided design model of the Adaptive Compliant Trailing Edge airplane.

The deflected ACTE flap during the ACTE aircraft research flights represents a change in the wing outer mold line, so it is important to understand the change in the aircraft aerodynamics produced by the ACTE flap throughout the entire flight envelope. In particular, the low-speed stall characteristics of the ACTE wing need to be analyzed and compared with the clean, unmodified wing in order to ensure flight safety for the take-off and landing phases of the ACTE aircraft.

We used the STAR-CCM $+{ }^{\circledR}$ (CD-adapco [Computational Dynamics Limited, United Kingdom, and Analysis \& Design Application Co., United States]) polyhedral unstructured computational fluid dynamics (CFD) code ${ }^{4}$ to conduct the wing stall analysis for an isolated ACTE wing on a symmetry plane at various ACTE flap deflections both in free air and in ground effect. The CFD methodology is next described. Then the effect of grid densities on the accuracy of the CFD wing stall predictions are quantified. Finally, low-speed stall characteristics of the ACTE wings are presented and compared with the clean wing.

\section{Computational Fluid Dynamics Methodology}

The STAR-CCM $+{ }^{\circledR}$ CFD code has been validated for high-lift wing aerodynamics analysis ${ }^{5}$ using the wind-tunnel test data and CFD results made available during the First American Institute of Aeronautics and Astronautics (AIAA) 
High-Lift Prediction Workshop. ${ }^{6}$ Reference 5 showed that the STAR-CCM $+{ }^{\circledR}$ CFD code can predict wing stall for the AIAA wing-body geometry to within $1 \mathrm{deg}$ AoA as compared to benchmark wind-tunnel test data.

At the un-deflected angle of $0 \mathrm{deg}$, the ACTE wing was designed to have the same outer mold line as a clean G-III wing. Therefore we call the 0-deg ACTE wing the clean wing hereafter, and use the clean wing CFD results as our baseline in the comparisons in this work. All of the CFD simulations in this work used the aircraft flight condition of $120 \mathrm{kn}$ and $2300 \mathrm{ft}$ above sea level (ASL), which is approximately ground-level at Edwards Air Force Base.

Figure 2 shows the mesh geometry. Only one wing on a symmetry plane was analyzed to efficiently resolve the wing stall flow physics. The aircraft fuselage and landing gear were not included in the present CFD analysis. Therefore the effects of these aircraft parts on the wing stall characteristics cannot be determined in the present study. As labeled in Fig. 2, the symmetry plane is brown, the ground plane is grey, the rotating overset mesh is the threedimensional green potato-shaped region immediately surrounding the wing, and the overlap mesh region is the fine mesh area that is bounded by the darker rectangular shapes on the ground and the symmetry planes. The overlap mesh region covers both the overset mesh and the surrounding background mesh and is used to ensure that the grid densities in the background and overset mesh regions match for the best solution accuracy. The overlap meshing region is part of the background mesh and thus remains stationary.

Hereafter, the angle of attack (AoA) is defined to be the aircraft AoA. In free air, the AoA is changed by simply changing the angle of the freestream velocity vector at the outer CFD far field boundary. Because of the presence of the ground plane in the ground effect case, simply changing the AoA of the freestream flow does not work. Instead, the entire overset mesh including the ACTE wing grid is rotated about the origin of the aerodynamic moment reference frame of the airplane to change the AoA value while the nearby ground is held fixed in the background mesh region.

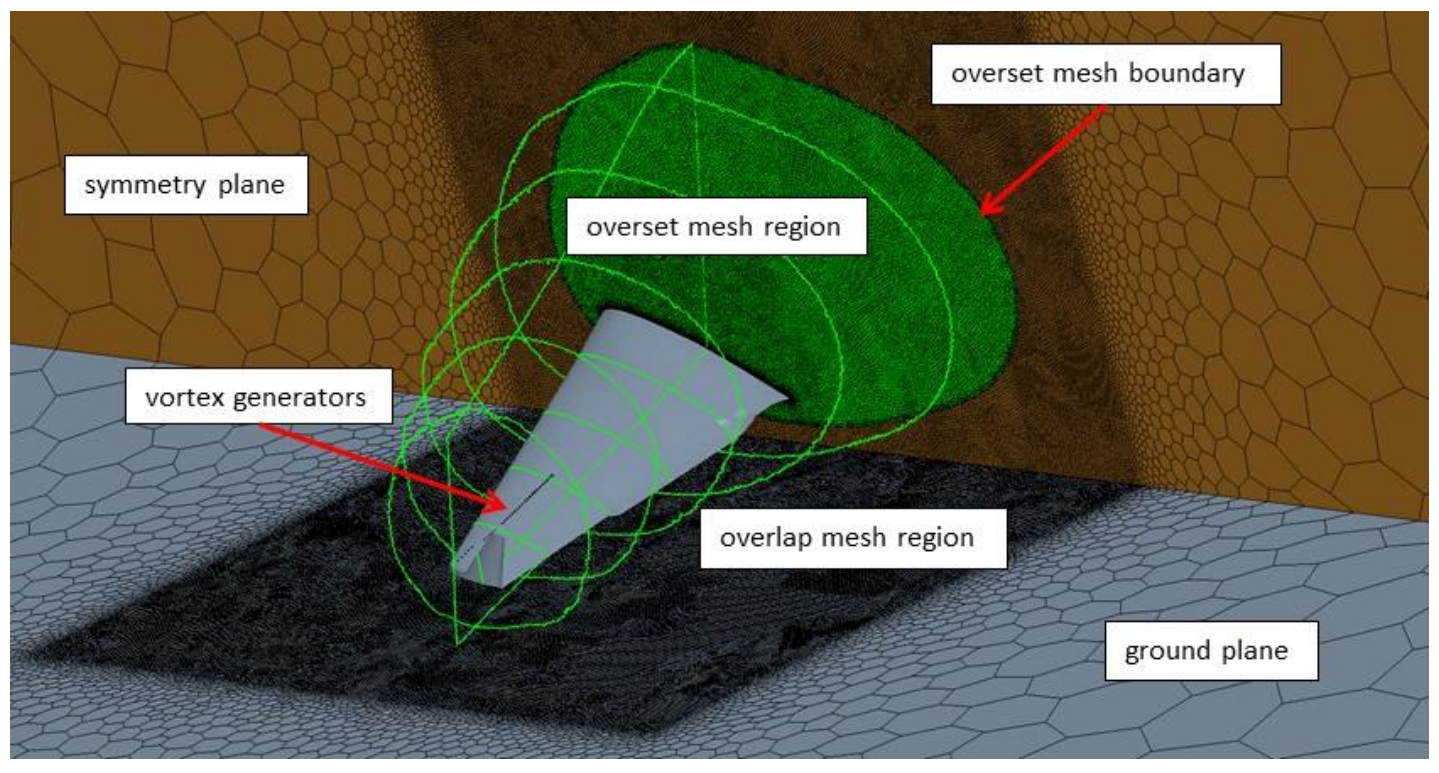

Figure 2. Computational fluid dynamics overset unstructured polygonal mesh for the 30-deg Adaptive Compliant Trailing Edge wing in ground effect.

It has been found that ground effect increases with lower aircraft height above ground. ${ }^{7}$ As the wing gets closer to the ground, it is stalled at a lower AoA value. The current wing stall analysis in ground effect was performed for the lowest possible height of the aircraft on the ground with the main landing gear strut fully compressed in order to provide results for a worse-case scenario. Inviscid wall boundary condition was used for the ground plane. Inviscid wall is the appropriate CFD boundary condition for the ground plane, since during take-off and landing the ground would be physically moving relative to the airplane wing at the same speed as the incoming air flow, assuming no wind.

The 31 vortex generators on the standard G-III aircraft wing are included in the present CFD model and shown in Fig. 2 as straight rows of small blades on the upper surface of the wing just inboard of the winglet. The CAD models for the vortex generators were constructed from actual measurements on our ACTE aircraft. 
Fourteen CFD cases are summarized in table 1 below. The CFD simulations were conducted for the -2-, 0- (clean wing), 15-, and 30-deg ACTE flaps in free air, as well as the 0- (clean wing), 15-, and 30-deg ACTE flaps in ground effect. For each configuration, a medium mesh (39.2 to 50 million cells) and a fine mesh (60.7 to 76 million cells) were used to evaluate grid convergence. Unstructured polyhedral meshes were used together with prism layers to model the near-wall boundary layer flows. As well, 19 and 23 prism layers were used in the near-wall region for the medium and fine meshes, respectively, for all wings. The prism layer is clustered near wall using the hyperbolic tangent stretching function. A total prism layer thickness of 2.2 in was found to provide appropriate coverage for the boundary layer region over the entire wing. The next-to-the-wall prism layer spacing of $7.9 \times 10^{-5}$ in. provides an average near-wall $\mathrm{y}^{+}$value of under 0.2 over most of the wall surfaces on the wing ranging from high values of approximately 0.4 near the leading edge to low values of approximately 0.05 near the trailing edge. Both the total prism layer thickness and the next-to-the-wall prism layer spacing were kept constant for the different mesh densities as well as different ACTE wing geometries. The Spalart-Allmaras one-equation turbulence model was used with low- $\mathrm{y}^{+}$wall treatment (no turbulence wall functions). Second-order Roe flux difference splitting was used with a coupled implicit flow solver.

Table 1. Computational fluid dynamics run cases and mesh densities.

\begin{tabular}{|l|c|c|c|}
\hline Case number & ACTE deflection, deg & Flow/mesh density & Mesh size, millions \\
\hline 1 & Clean wing & Free air/medium & 45.1 \\
\hline 2 & Clean wing & Free air/fine & 61.9 \\
\hline 3 & -2 & Free air/medium & 47.3 \\
\hline 4 & -2 & Free air/fine & 63.0 \\
\hline 5 & 15 & Free air/medium & 47.5 \\
\hline 6 & 15 & Free air/fine & 63.0 \\
\hline 7 & 30 & Free air/medium & 48.9 \\
\hline 8 & 30 & Free air/fine & 64.6 \\
\hline 9 & Clean wing & Ground effect/medium & 39.2 \\
\hline 10 & Clean wing & Ground effect/fine & 60.7 \\
\hline 11 & 15 & Ground effect/medium & 50.6 \\
\hline 12 & 15 & Ground effect/fine & 75.3 \\
\hline 13 & 30 & Ground effect/medium & 46.3 \\
\hline 14 & 30 & Ground effect/fine & 76.0 \\
\hline
\end{tabular}

The following steps were used for each ACTE wing geometry to make a complete AoA sweep and obtain the wing's lift curve through maximum lift:

1) Start the AoA sweep with a CFD simulation of the wing at a small initial AoA value. Uniform freestream flow could be used as the initial condition for the first CFD simulation of the sweep. The starting AoA should be well below the AoA value near maximum lift. Starting the sweep at too high an AoA value could trigger unphysical flow separation and premature wing stall. For the current work, a starting AoA value at approximately $6 \mathrm{deg}$ is appropriate.

2) Once the CFD solution for the lower AoA value converges, increase the AoA to the next higher value by either incrementing the AoA of the freestream velocity at the far field CFD boundary for the free-air case, or rotating the wing overset mesh region for the ground effect case. An AoA increment value of approximately $2 \mathrm{deg}$ is found to work well at lower AoA ranges.

3) Restart the CFD simulation at the higher AoA value from the previously converged, lower AoA CFD solution.

4) Repeat steps 2 and 3 above until the wing lift is lost in the CFD solution, indicating wing stall has been reached. It is likely that the CFD wing stall occurs prematurely during the sweep due to a larger AoA increment value than required. It is inefficient to use a very small AoA increment from the beginning of the AoA sweep - the number of the CFD simulations required would be more than necessary. Instead, proceed with step number 5 below.

5) Repeat steps 2 and 3 from the last maximum lift solution, but with an AoA increment that is half as large as the previous AoA increment, for example $1 \mathrm{deg}$, until the wing stall AoA is reached again. 
6) Repeat step 5 above from the latest maximum lift solution for a yet smaller increment of AoA, for example $0.5 \mathrm{deg}$, if required. The wing stall solution obtained with the smallest AoA increment is then used as the final solution. The minimum AoA increment used for the current work is $0.5 \mathrm{deg}$.

The final AoA increment can be used as an indication of the uncertainty in the predicted wing stall AoA value. It is not possible to predict the wing stall AoA value with an uncertainty that is less than the smallest AoA increment that was used during the sweep.

\section{Adaptive Compliant Trailing Edge Wing Stall Computational Fluid Dynamics Results}

\section{A. Computational Fluid Dynamics Grid Convergence Studies}

Studies were made to evaluate grid convergence of the CFD solutions, both for the free air as well as ground effect cases. Figure 3 plots the grid convergence results for the wing in free-air case with the -2-deg, clean, 15-deg, and 30-deg ACTE wings. The medium grid results with the circle symbols agree well with the fine grid results with the $\times$ symbols for all of the lift curves. Therefore, grid convergence is generally reached, except for the discrepancies in the exact locations of maximum lift. The fine mesh results generally predict maximum lift occurring approximately 0.5 to 1.0 deg higher in AoA than the medium mesh results for all wings. Note that this is within the 1 deg of uncertainty as observed in reference 5 .

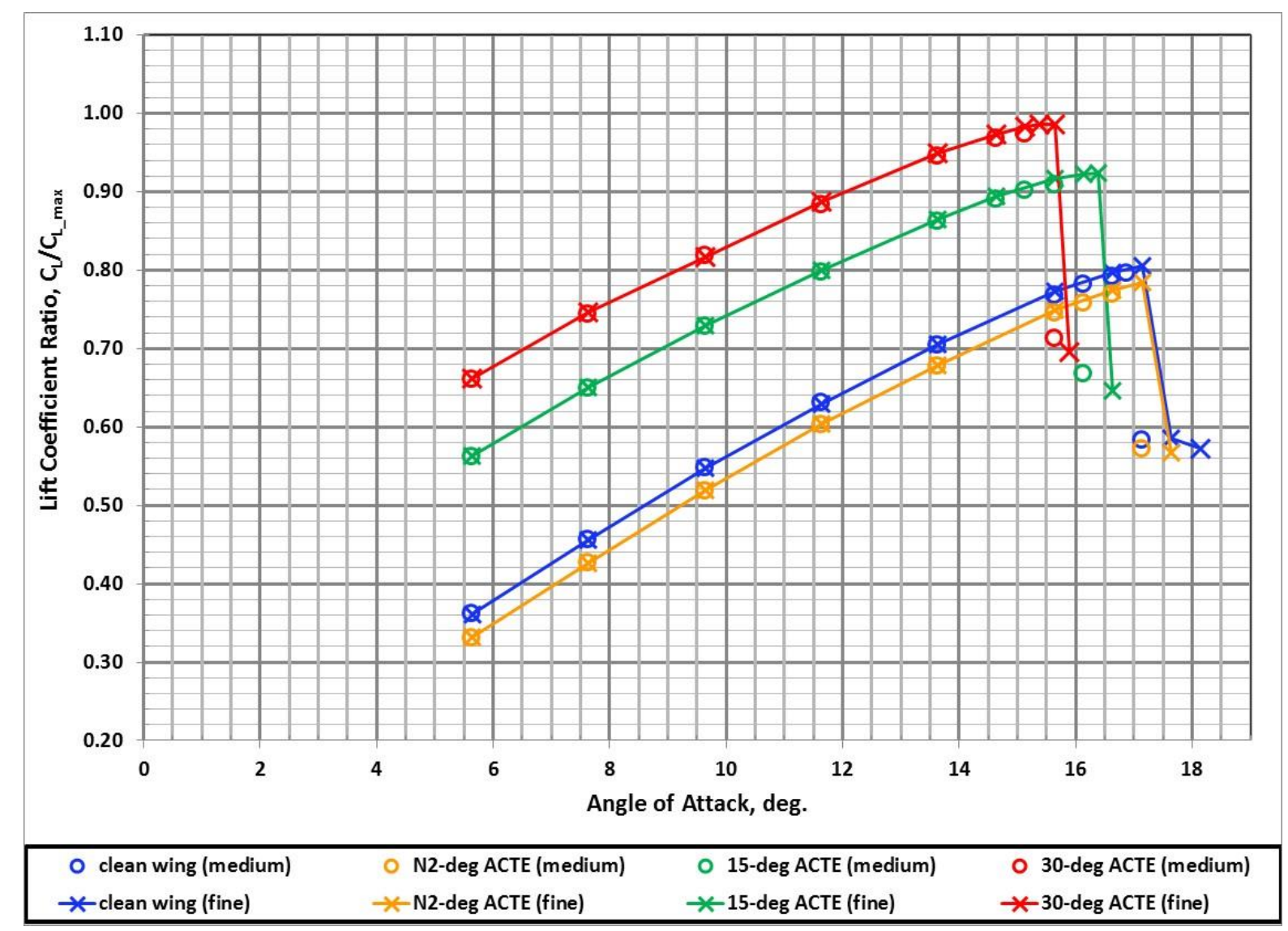

Figure 3. Computational fluid dynamics grid convergence results for the Adaptive Compliant Trailing Edge wing in free air.

Figure 4 plots the grid convergence results for the wing in the ground effect case with the clean, 15-deg, and 30-deg ACTE wings. As can be seen from Fig. 3 above, the aerodynamic stall characteristic of the -2-deg ACTE wing is very similar to that of the clean wing: both wings stall at approximately AoA $=17 \mathrm{deg}$. Therefore we do not analyze the -2-deg ACTE wing in ground effect, and we make the assumption that the -2-deg ACTE wing has the same stall 
characteristics as the clean wing in ground effect. The grid convergence for the ground-effect case is actually better than that of the wing in the free-air case. This is probably due to the additional mesh refinement in the space between the wing and the ground plane. Since the free-air cases did not contain the ground plane, there was no mesh refinement done in the space below the wing. Very good agreements are obtained between the clean wing and the 15-deg ACTE wing. For the 30-deg ACTE wing, the finer mesh predicts wing stall at an AoA value approximately 0.5 deg higher than the medium mesh.

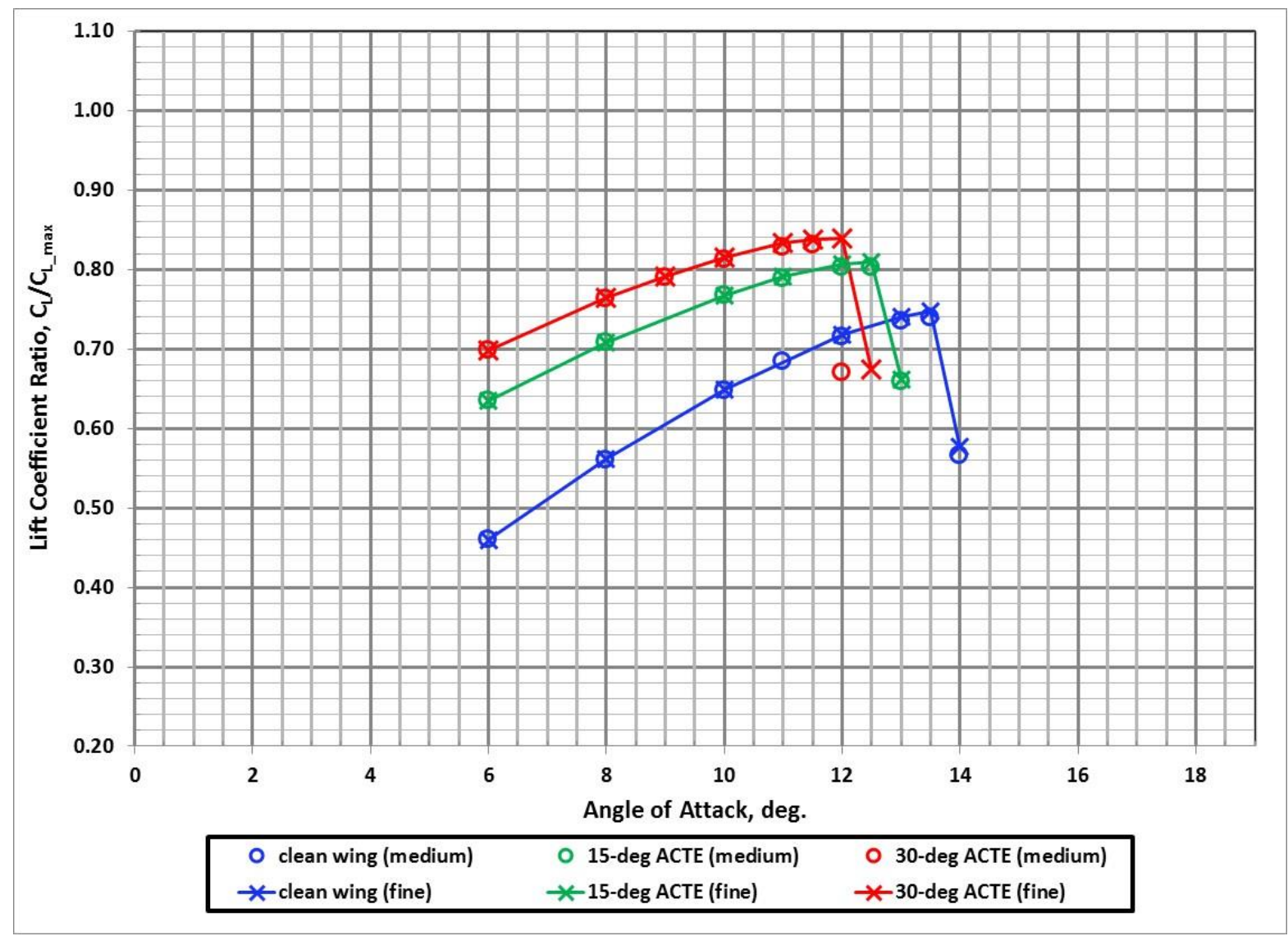

Figure 4. STAR-CCM $+{ }^{\circledR}$ computational fluid dynamics grid convergence results for the Adaptive Compliant Trailing Edge wing in ground effect.

\section{B. Wing Stall Computational Fluid Dynamics Results}

Figure 5 presents the CFD wing stall results for the ACTE wing. Fine mesh results from the free-air as well as ground-effect cases are shown. The clean wing results are colored blue; the 15-deg results are green; and the 30-deg results are red. The dashed lines are the free-air results, and the solid lines are the ground-effect results. In free air, it can be seen that the 15-deg ACTE wing produces higher lift and stalls at approximately 0.5 deg lower in AoA than the clean wing. Also in free air, the 30-deg ACTE wing stalls at approximately 1.5 deg lower in AoA than the clean wing. 


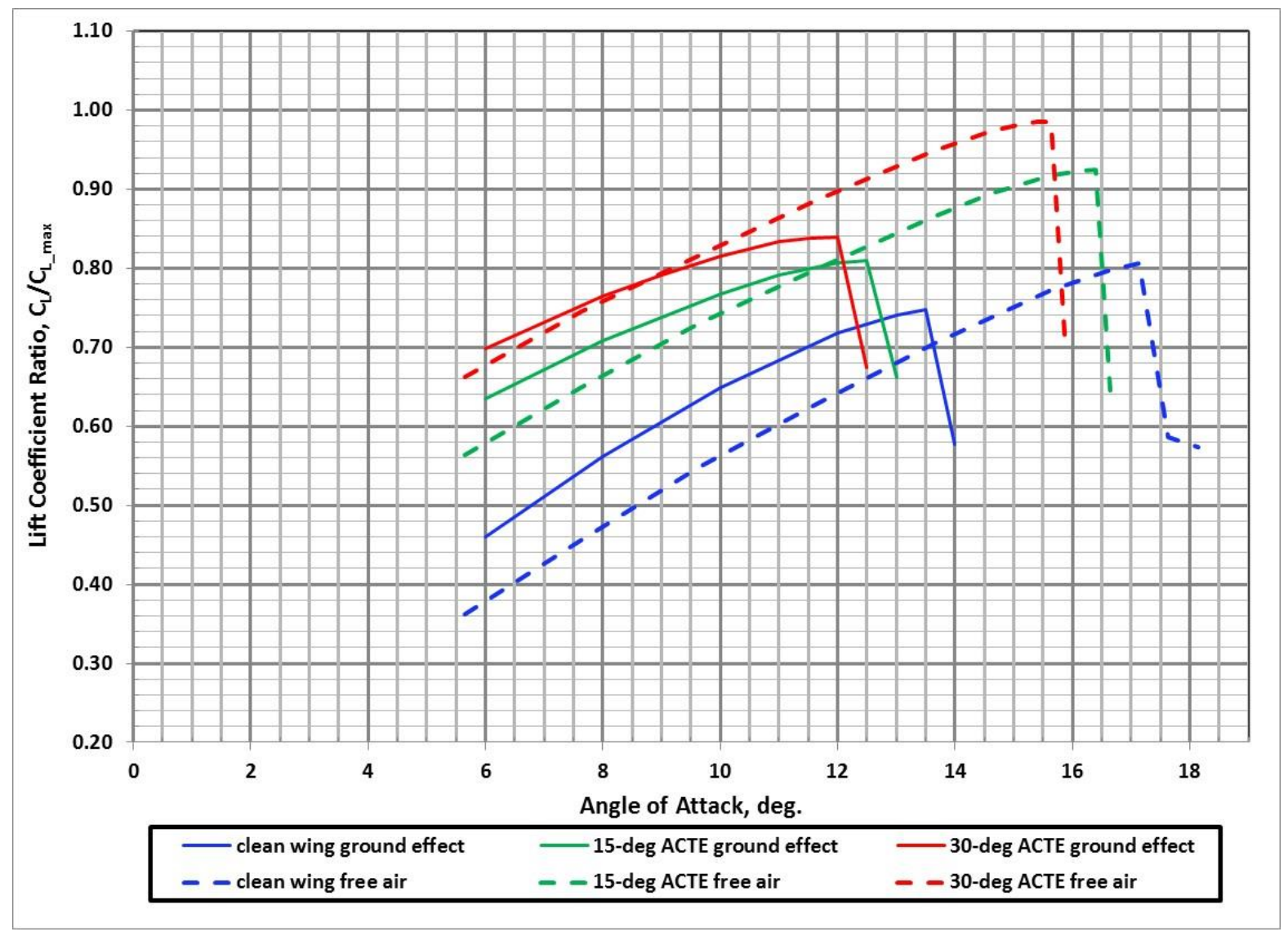

Figure 5. STAR-CCM $+{ }^{\circledR}$ computational fluid dynamics comparison between free air and ground effect stall results for the Adaptive Compliant Trailing Edge wing - fine grid results only.

Ground effect is known to cause aircraft wing stall at lower AoA values and could lower the maximum wing lift coefficient ${ }^{7}$. It can be seen in Fig. 5 that all wings in ground effect produce smaller maximum lift coefficient values than the corresponding wings in free air. Ground effect significantly raises the lift curve for the clean wing. The solid blue line, clean wing in ground effect, is much higher than the dashed blue line, clean wing in free air. Ground effect raises the lift curves less for the 15-deg and the 30-deg ACTE wings as compared to the clean wing. In addition, the ACTE wing lift curves in ground effect, the green and red solid lines, actually dip below their corresponding curves in free air, the green and red dashed lines, at higher AoA's. The clean wing lift curve in ground effect, blue solid line, stays higher than the corresponding free air lift curve, the blue dashed line, all the way to maximum lift. In all cases, wing stall is characterized by a sharp break at maximum lift coefficient with the ACTE wings exhibiting a stall characteristic similar to that of the clean wing.

Flow visualizations of the flow physics over the clean wing at $\mathrm{AoA}=13 \mathrm{deg}$, the 15-deg ACTE wing at $\mathrm{AoA}=12.5 \mathrm{deg}$, and the 30-deg ACTE wing at $\mathrm{AoA}=12.5 \mathrm{deg}$ are shown in figures 6(a), 6(b), and 6(c), respectively. Since the clean wing is still far from maximum lift at this AoA value, larger AoA increments were used, and the 12.5-deg AoA solution is not available for the clean wing. All of these results are in ground effect. Free air results exhibit similar trends. In these figures, 3D views of the 3D wings are shown in the left, and 2D cross-sectional cuts at a fixed spanwise wing station are shown in the right. The cross-sectional cut's spanwise location on the 2D views on the right is indicated by the thick blue lines on the wing and the ground plane in the 3D views on the left. The blue elliptical outlines seen in both the right and left sides are the boundary of the overset mesh region which encloses the wing as discussed in the section "Computational Fluid Dynamics Methodology" above. In the 3D views on the left, flow streamlines over the wing are plotted together with contours of pressure coefficients on the wing, symmetry 
plane, and ground plane surfaces. In the 2D cross-sectional cuts on the right, the flow streamlines and Mach number contours are shown.

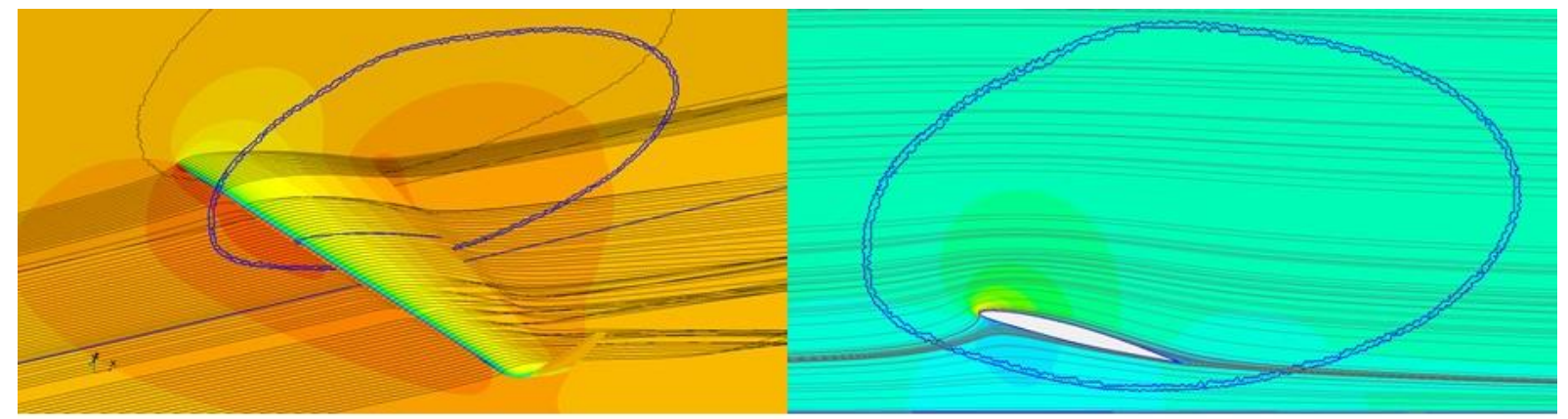

(a) Clean wing; $\mathrm{AoA}=13 \mathrm{deg}$

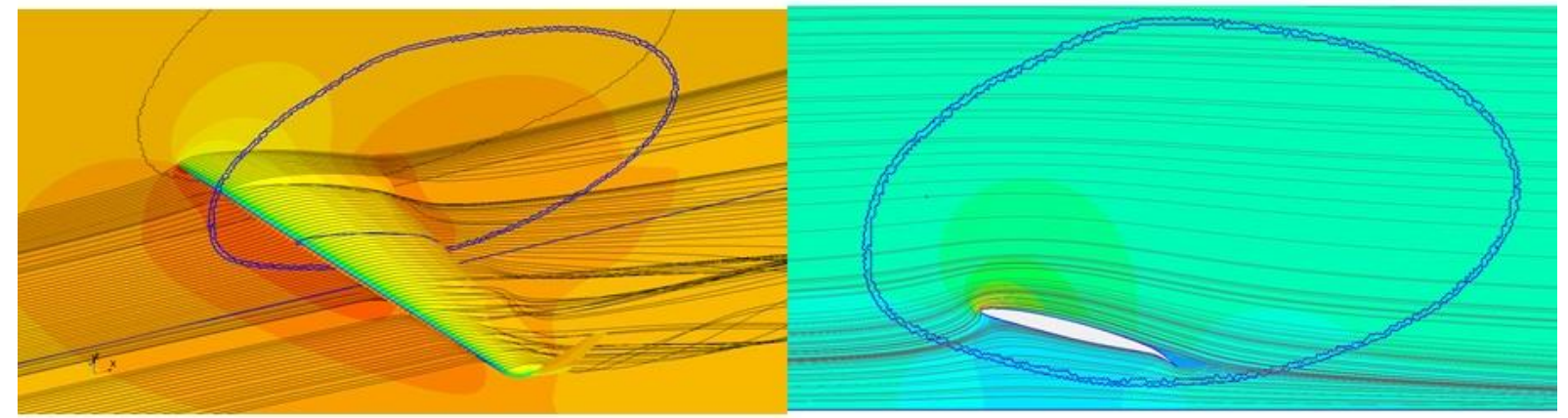

(b) 15-deg ACTE; AoA = $12.5 \mathrm{deg}$

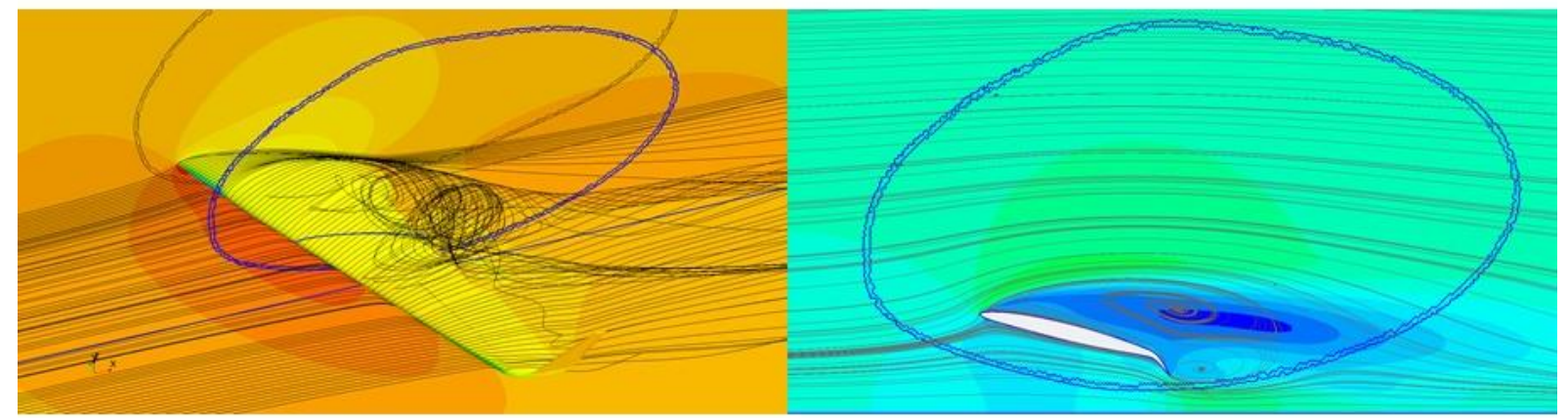

(c) 30-deg ACTE; AoA = $12.5 \mathrm{deg}$

Figure 6. Ground effect flow visualization results for the Adaptive Compliant Trailing Edge flap wings.

It can be seen that the flow over the 30-deg ACTE wing is stalled earlier than both the clean wing and the 15-deg ACTE wings. A large flow separation region is located right above the ACTE flap area. As can be seen on the left side of Fig. 6c, the stalled flow separation bubble over the wing is in the same approximate spanwise location as the 30-deg ACTE flap. The 2D contour plot on the right side of Fig. 6c confirms the existence of this large flow separation bubble extending over the entire airfoil chord from the leading edge to the trailing edge. Therefore, the ACTE flap is predicted to be the cause of the premature wing stall. Although the 15-deg ACTE wing is not yet stalled, the 2D contour plot on the right of Fig. 6(b) shows a small flow separation bubble on the upper surface of the trailing edge. Finally, even though it is at a slightly higher AoA than the ACTE wings the flow over the clean wing is nice and smooth with no flow separation. 


\section{Conclusion}

Computational fluid dynamics (CFD) analysis was conducted to study the low-speed stall characteristics of a G-III aircraft swept wing modified with a seamless compliant Adaptive Compliant Trailing Edge (ACTE) flap at various ACTE deflection angles in free air and in ground effect using the STAR-CCM+ ${ }^{\circledR}$ (CD-adapco [Computational Dynamics Limited, United Kingdom, and Analysis \& Design Application Co., United States]) CFD code. The ACTE wing was analyzed at various flap deflections and compared to a clean wing. It was found that the ACTE wing is stalled at an earlier angle of attack than the clean wing. The ground plane decreased the stall angle of attack for all wings. Ground effect also decreased the maximum wing lift coefficient values for all wings as compared to the corresponding free air values. Higher ACTE flap deflections were seen to have smaller increases in lift due to ground effect. In particular, the ACTE wing is predicted to have less lift in ground effect than in free air at higher angle of attack values near maximum lift. Flow visualizations showed that the post-stall flow separation bubble is directly over the ACTE flap covering essentially the full chord of the wing, indicating that the ACTE flap is likely the cause for earlier ACTE wing stall as compared to the clean wing.

\section{References}

${ }^{1}$ Baumann, E., Hernandez, J., and Ruhf, J., "An Overview of NASA's SubsoniC Research Aircraft Testbed (SCRAT)," AIAA-2013-5083, 2013.

${ }^{2}$ Kota, S., Flick, P., and Collier, F., "Flight Testing of the FlexFoil ${ }^{\mathrm{TM}}$ Adaptive Compliant Trailing Edge," AIAA-2016-0036, 2016.

${ }^{3}$ Kota, S., Osborn, R., Ervin, G., Maric, D., Flick, P., and Paul, D., "Mission Adaptive Compliant Wing - Design, Fabrication, and Flight Test," RTO-MP-AVT-168, pp.18-1-18-19.

${ }^{4}$ Hanke, J., Shankara, P., and Snyder, D., "Numerical Simulation of DLR-F11 High Lift Configuration from HiLiftPW-2 using STAR-CCM+," AIAA-2014-0914, 2014.

${ }^{5}$ Bui, T. T., "Analysis of Stall Aerodynamics of a Swept Wing with Laminar-Flow Glove," Journal of Aircraft, Vol. 52, No. 3, May-June 2015, pp. 867-871.

${ }^{6}$ Rumsey, C. L., Slotnick, J. P., Long, M., Stuever, R. A., and Wayman, T. R., "Summary of the First AIAA CFD High-Lift Prediction Workshop," Journal of Aircraft, Vol. 48, No. 6, November-December 2011, pp. 2068-2079.

${ }^{7}$ O'Callaghan, J., "Aircraft Performance Study," National Transportation Safety Board Office of Research and Engineering, Washington, D.C., NTSB \# DCA11MA076, 2012. 